\title{
Instrumento evaluador de la credibilidad emprendedora en estudiantes de educación postobligatoria
}

\author{
Marc Mussons-Torras y Elisenda Tarrats-Pons \\ Universitat de Vic-Universitat Central de Catalunya
}

\section{RESUMEN}

El artículo describe el proceso de elaboración y validación de un cuestionario que analiza las dimensiones sobre la credibilidad emprendedora a 84 estudiantes que cursan formaciones de nivel postobligatorio. En primer lugar, se ha analizado la validez de contenido mediante un juicio de expertos, la validez de constructo mediante el análisis factorial, y finalmente la fiabilidad a través del Alfa de Cronbach. Los resultados muestran que el cuestionario es válido, con índices de fiabilidad suficientes, permitiendo disponer de un instrumento evaluador de la credibilidad emprendedora en relación con las variables cualitativas nominales dicotómicas, policotómicas y ordinales según escala sumativa.

Palabras Clave: credibilidad emprendedora, emprendimiento, formación post obligatoria, rasgos de personalidad.

\section{Evaluation instrument to the entrepreneurial student's credibility on the non-compulsory education}

\section{ABSTRACT}

This paper describes the process of development and validation of a questionnaire that analyzes the dimensions on entrepreneurial credibility to 84 students from non-compulsory education. First, we analyzed the content validity by an expert judgement. After that, the construct validity using factor analysis. And finally, the reliability through Cronbach's alpha. The results show that the questionnaire is valid, with sufficient reliability, allowing to have an instrument to evaluate entrepreneurial credibility in relation to the dichotomous and polychotomous nominal qualitative variables and to the ordinal qualitative variables according summative scaling.

Keywords: entrepreneurial credibility, entrepreneurship, non-compulsory education, personality.

\section{Introducción}

Desde el año 2008, la mayoría de las economías del mundo entraron en recesión. Y aunque se constata que hay signos de mejoría, aún no son lo suficientemente significativos como para poder afirmar que nos encontramos en la antesala de un nuevo cambio de ciclo económico. Esta falta de crecimiento económico tiene sus consecuencias en elevadas tasas de desempleo, especialmente preocupantes en el caso de los más jóvenes. La tasa de desempleo juvenil en los países europeos del arco del mediterráneo agudiza aún más el problema. La tasa de desempleo juvenil de los países de Grecia (50,7\%), España (53,9\%), Italia (42,9\%) y Portugal (35,5\%), encabezan la lista entre todos los países de la Unión Europea (Eurostat regional yearbook 2014, n.d.).

El Espacio Europeo de Educación Superior (EEES), en la Declaración de Bolonia (Eurostat, 2009) establece el objetivo de creación de un sistema universitario donde los titulados puedan hacer frente a la demanda de un mercado laboral cada vez más internacional, con la investigación orientada en la innovación, siendo el motor dinámico para la evolución tecnológica, económica, social y cultural de la nueva sociedad.
Asimismo, el actual proceso de globalización exige nuevas competencias a los empleados que los sistemas educativos deben proveer para una adecuada incorporación laboral de los mismos. La globalización tiene implicaciones directas en la educación superior, concretamente por la creciente importancia de la economía vinculada con la sociedad del conocimiento, la innovación y la relevancia de todos estos procesos en un contexto donde el mercado global adopta un rol central (UNESCO, 2004).

Según la Comisión Europea, hay dos áreas principales de actividad que las universidades deberán potenciar: La primera, la calidad de la investigación, prioridad establecida en la Estrategia de Lisboa (Consejo Europeo, 2000) con el objetivo de hacer de la UE una de las economías más competitivas y dinámicas del mundo en lo que se refiere al concepto de "sociedad del conocimiento". Y la segunda la innovación, entendida como la ejecución de procesos e ideas que permitan conectar la investigación universitaria con las actividades empresariales y de desarrollo económico. La estrategia Europa 2020 establecida por la Comisión Europea, busca garantizar el crecimiento de forma inteligente, sostenible y de responder a estas necesidades mediante la reforma del sistema de educación superior, en un momento en 
que la economía europea está expuesta a debilidades estructurales y sometida a una larga recesión económica y financiera.

En este nuevo escenario de cambio, la innovación y la competitividad están directamente asociadas con la capacidad de las universidades de reaccionar de forma rápida y eficiente a las demandas de la sociedad, y en particular del mercado. La Unión por la innovación, engloba directrices y políticas de la Comisión Europea para salir de la recesión, centradas en promover el espíritu emprendedor, apoyando a jóvenes empresas innovadoras, y reforzando los vínculos entre educación, empresa, investigación e innovación. La Comisión Europea en la estrategia Europa 2020, y en línea con el tratado de Bolonia y la estrategia de Lisboa, refuerza la cooperación entre universidad, investigación y empresa para dar el salto en I+D+i necesario (crecimiento inteligente) con la finalidad de dejar atrás el largo periodo de recesión.

Uno de los aspectos fundamentales para que la universidad se adapte con éxito a la nueva sociedad del conocimiento es que ésta promueva y adopte nuevos roles y actividades lejos de sus tradicionales funciones de formación e investigación. Entre ellos destacamos el fomento del espíritu emprendedor, la transferencia de conocimiento, la formación continua y la innovación, así como la creación de vínculos cada vez más fuertes entre universidad y empresa que deberán servir para que la universidad lidere nuevas funciones de éxito que supongan un mayor impacto económico, social y cultural (Reichert, 2009).

Existen diferentes modelos que profundizan en la comprensión del espíritu emprendedor que impulsa a un individuo en un determinado momento a convertirse en auto empleado y fundador de su propia empresa. Entre ellos destacamos el "modelo del potencial emprendedor" desarrollado por Krueger y Brazeal (1994). Este modelo incorpora los modelos previos del evento emprendedor (Shapero y Sokol, 1982), y la teoría del comportamiento planificado (Ajzen, 1991), integrando los conceptos de percepción de deseabilidad y de viabilidad.

En el Modelo citado, la percepción de deseabilidad hace referencia al propio deseo de emprender. Un deseo basado en una expectativa de ganancia económica, pero también de éxito social y de reconocimiento. En la percepción de deseabilidad del sujeto, también le inciden lo que pensarán las personas más cercanas e importantes de su vida, sobre la posibilidad de que constituya una nueva empresa.

En este sentido destacamos las normas sociales de su entorno, denominadas normas subjetivas, dado que la opinión colectiva de la propia sociedad y del entorno próximo del sujeto puede ser relevante para él. La percepción de viabilidad, en cambio, se refiere a la habilidad personal para desempeñar eficazmente un comportamiento emprendedor, donde el individuo lo percibe como viable. En este modelo la credibilidad es un factor clave para que el comportamiento sea considerado necesario y viable, lo cual influirá en la intención de crear una empresa. Por tanto, la credibilidad juntamente con la propensión a actuar, determinarán el potencial emprendedor. El modelo también explica que, aunque una persona pueda percibir que la creación de una empresa es deseable y viable, puede que no tome la decisión por falta de un suceso disparador. El potencial emprendedor se transforma en intención siempre y cuando aparezca un suceso disparador. Este suceso disparador se concibe como aquel cambio trascendental del sujeto que rompe la inercia de su carrera profesional y le impulsa en su decisión de crear una empresa.

En relación con el desarrollo de instrumentos de medición de la credibilidad emprendedora tienen su origen en los países anglosajones siendo sus máximos exponentes: el test EAO -Entrepreneurial Attitude Orientation- desarrollado por Robinson et al. (1991), el test ATE - Attitudes Toward Enterprise- (Athayde, 2009), el test EIQ - Entrepreneurial Intention Questionnaire- de los autores Liñan y Chen (2009) y el CDQ -Choice Dilemmas Questionare- de Brockhaus (1980). Acorde a estos instrumentos de medida de la credibilidad emprendedora y a los principales test de personalidad: NEO-PIR (Costa y Mc Crae, 2002), EPQ (Eysenck y Eysenck, 1994a), 16 PF (Russell et al., 1997a), I-E (Rotter, 1966), la escala de automonitoreo (Lennox y Wolf, 1984) y el ensayo sobre las mediciones de las actitudes sociológicas sociales (Robbins, 1996) desarrollamos un cuestionario de medida de la credibilidad emprendedora ajustado al perfil de los jóvenes españoles.

\section{Método y procedimiento}

\section{Objetivos}

La investigación pretende medir las dimensiones y los factores que inciden en la credibilidad emprendedora de los estudiantes de educación post obligatoria que cursan bachillerato, ciclo formativo de grado superior y graduado universitario. Para ello, el presente estudio analiza todo el proceso de construcción y validación de un instrumento evaluador con los siguientes hitos:

1) Analizar la validez de contenido y de constructo del instrumento.

2) Examinar la fiabilidad del instrumento.

\section{Construcción}

El cuestionario se ha construido ad-hoc, utilizando ítems procedentes de las investigaciones científicas de los autores más relevantes. La variable dependiente, denominada "credibilidad emprendedora", está formada por ítems basados en la escala de actitudes de orientación emprendedora de los autores Robinson, Stimpson Huefner y Hunt (1991), siendo asimismo utilizados por Singh y DeNoble (2003) (ítems no 7, 9 y 11).

Por su parte, las variables independientes se agrupan en dimensiones de la percepción de deseabilidad y de la percepción de viabilidad. En percepción de deseabilidad se incluyen los factores sociodemográficos y los factores de personalidad. En percepción de viabilidad se incluyen los factores económicos de tipo exógeno y los factores personales o de tipo endógeno. En la Figura 1 se reflejan las diferentes dimensiones analizadas para determinar la credibilidad emprendedora, a saber:

Figura 1. Dimensiones y factores de la credibilidad emprendedora. Fuente: Elaboración propia.
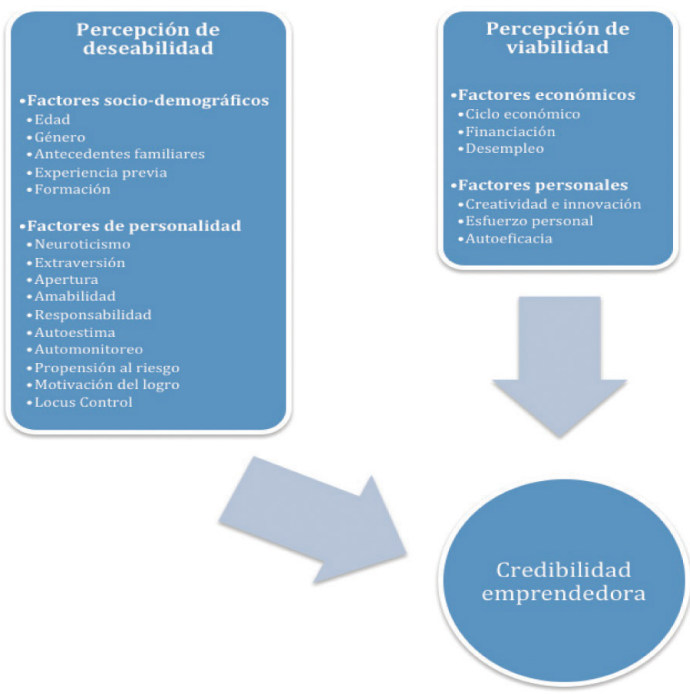
Como puede observarse, la variable nominal dicotómica de género se añade en el cuestionario por el elevado contraste y significación de dicha variable en relación a la variable dependiente. Siendo el género masculino más tendente al emprendimiento que el femenino por connotaciones sociales y cargas familiares (Athayde, 2009; Chen, Greene, y Crick, 1998; Crant, 1996; Jose, Liñan, y Santos, 2009; Kolvereid, 1996; Liñán, Santos, y Fernández, 2011; Veciana, Aponte, y Urbano, 2005; Wang y Wong, 2004). Lo mismo sucede con los antecedentes familiares donde la valoración fuerte de vínculo es decir tener familiares empresarios es determinante para influenciar en la credibilidad emprendedora (Athayde, 2009; Carayannis, Evans, y Hanson, 2003; Crant, 1996; Espí, Arana, Heras, y Diaz, 2007; Veciana et al., 2005; Wang y Wong, 2004; Wang, Lu, y Millington, 2011). Y también el apoyo de amigos empresarios (Honig y Davidsson, 2003).

En relación a la experiencia laboral previa, el hecho de haber trabajado o estar trabajando incide también en mayores niveles de credibilidad emprendedora (Honig y Davidsson, 2003; Peterman y Kennedy, 2003; W. Wang et al., 2011).

Por otro lado, lo mismo sucede con el nivel de formación, advirtiéndose que ndo a mayor nivel formativo, mayor credibilidad emprendedora (Chen et al., 1998; Crant, 1996; Honig y Davidsson, 2003; Linan, Urbano y Guerrero, 2011; Robinson et al., 1991). Especialmente en la formación sobre creación de empresas y sobre el emprendimiento en todas las edades y con enfoques más prácticos que teóricos (Carayannis et al., 2003; Drost, 2010; Dyer, 1994; Eldelman, Manolova, y Brush, 2008; Henry, Hill, y Leitch, 2005; Hisrich y Peters, 2002; Honig, 2004; Krauss, 2011; Krueger Jr y Brazeal, 1994; Lepoutre, Berghe, Tilleuil, y Crijns, 2010; Piaget, 1995; Robinson et al., 1991; Rusell, Atchison, y Brooks, 2008; Souitaris, Zerbinati, y Al-Laham, 2007; Thursby, Fuller, y Thursby, 2009).

En la construcción de los ítems ordinales que detallan los factores de la personalidad del sujeto se ha utilizado una escala sumativa de Likert (1932) de cuatro posiciones para evitar la respuesta neutral.

En la dimensión de neuroticismo se han añadido sus cinco subescalas: Ansiedad, Hostilidad, Depresión, Ansiedad Social e Impulsividad, y los ítems han sido seleccionados de los formularios del NEO PI-R de Costa y McCrae (2002), y del EPQ de Eysenck y Eysenck (1994b). Los autores destacan que el sujeto neurótico, y emocionalmente inestable, se inhibe del emprendimiento, siendo la correlación inversa con la credibilidad emprendedora (Singh y DeNoble, 2003).

En la dimensión extraversión se han añadido sus cinco subescalas: Cordialidad, Gregarismo, Asertividad, Actividad, Búsqueda de Emociones y Emociones Positivas. Es un rasgo que indica afectotímia que es propio de personas abiertas, afectuosas y participativas (Russell et al., 1997b) aunque también indica optimismo, excitación, riesgo y despreocupación (Eysenck, 1970). En el estudio de Singh y DeNoble (2003) se encontró una fuerte correlación entre extraversión e intención emprendedora en estudiantes universitarios.

En la dimensión apertura, menos estudiada que las dos anteriores, define a las personas abiertas, creativas, originales y receptivas a nuevas experiencias. Siendo su relación indirecta con el emprendimiento y directa con la innovación (Athayde, 2009; Ismail, Jaffar, y Siow, 2013; McCrae y Costa, 2003; Robinson et al., 1991; Singh y DeNoble, 2003). También se añaden al estudio sus cinco subescalas: Fantasía, Estética, Sentimientos, Acciones, Ideas y Valores (Costa y Mc Crae, 2002).

En la dimensión amabilidad en sus cinco subescalas de: Confianza, Franqueza, Altruismo, Actitud conciliadora, Modestia y
Sensibilidad a los demás (Costa y Mc Crae, 2002), se nos define al sujeto como un término medio entre dos extremos. Según Horney (1945), los extremos son de movimiento hacia la gente y de movimiento contra la gente. Para el primer extremo, encontramos a las personas dependientes y sumisas. En el otro extremo a las personas narcisistas, egoístas y antisociales. La relación entre el rasgo psicológico de amabilidad con el emprendimiento no es significativa y en muchos casos de signo contrario. Los niveles elevados de amabilidad suelen conllevar niveles altos de pasividad y dependencia; factores, todos ellos, que los tomadores de riesgo no deberían de poseer (Brice, 2002; Singh y DeNoble, 2003). En cambio, otros autores destacan la elevada correlación significativa con el desempeño académico, el trabajo en equipo, y el aprendizaje (Lounsbury, Gibson, Sundstrom, Wilburn, y Loveland, 2004).

En la dimensión de responsabilidad en sus cinco subescalas de: Competencia, Orden, Sentido del deber, Necesidad de Logro, Autodisciplina y Deliberación (Costa y Mc Crae, 2002) se define al sujeto como diligente, organizado, ordenado y persistente. Siendo una variable más relacionada con la gestión del negocio y con el rendimiento profesional que con el emprendimiento. El espíritu emprendedor va más relacionado con oportunidades de negocio, mentes creativas y menos estructurados de mente (Costa y Mc Crae, 2002; Singh y DeNoble, 2003).

Por otro lado, y en referencia a la variable autoestima entendida como la autoconfianza personal (self-esteem) es la capacidad de verse uno mismo capaz de superar los retos profesionales, siendo determinante para el éxito empresarial y el emprendimiento (Ismail et al., 2013; Krauss, 2011; Nandamuri, Gajulapa1ly, y Gowthami, 2012; Robinson et al., 1991). La autoestima actúa como un facilitador frente a los factores de riesgo del trabajo, razón por la cual es importante que el emprendedor posea una alta autoestima, capaz de resguardar la armonía y la dignidad en condiciones de presión y estrés (Harvey y Keashly, 2003). En definitiva, la autoestima es un estado de autoconfianza que se retroalimenta. Hace que las personas busquen objetivos más exigentes, y al lograrlos su autoestima aumenta aún más (Branden, 1993). Los ítems para evaluar la dimensión autoestima son extraídos del trabajo de A.H Eagly, en su adaptación de J.R Robinson y P.R Shaver (Robbins, 1996), y del propio cuestionario del EAO de Robinson et al. (1991).

El automonitoreo, o máscara social (Russell et al., 1997b) es la característica de la personalidad que mide la habilidad del individuo de ajustar su comportamiento a los factores situacionales externos. Según Robbins (1996) los individuos que puntúan alto en auto monitoreo son personas polivalentes en su carrera profesional a la vez que exitosos en puestos de trabajo de "administradores, gerentes o empresarios" donde se requiere la capacidad de desempeñar múltiples roles, incluso contradictorios, que obligan al individuo a adaptar su comportamiento ante diferentes públicos e intereses. Para la elaboración de las preguntas del cuestionario nos hemos basado en el estudio de Lennox y Wolf (1984) y su escala de auto monitoreo. Cabe decir que las preguntas de su test coinciden con las preparadas para la dimensión $\mathrm{N}$ de máscara social de la escala de los dieciséis factores de personalidad de Raymond B. Cattell (Russell et al., 1997b), en su versión para adultos $\mathrm{A}$.

La variable de propensión al riesgo se define como la preferencia por las situaciones que pueden aportar beneficios y recompensas en caso de éxito, pero también severas consecuencias en caso de fracaso (Brockhaus, 1980). Es más según Liles (1974), el riesgo de ser emprendedor se manifiesta también a nivel personal y emocional. Y especialmente en países como Francia donde el fracaso como emprendedor crea estigma social (Carayannis 
et al., 2003). O en Singapur, donde la aversión al riesgo vienen condicionada por su coste de oportunidad entendido como la facilidad de encontrar un trabajo más seguro y bien remunerado en alguna de las grandes corporaciones multinacionales (Wang y Wong, 2004). En cualquier caso, cierto es que se considera el riesgo como uno de los principales frenos al emprendimiento (Espí et al., 2007). Y que la variable propensión al riesgo correlaciona con la intención emprendedora entre los estudiantes universitarios (Chen et al., 1998; Lüthje y Franke, 2003; Wang et al., 2011). En el cuestionario se incorporan los ítems según el formulario CDQ - Choice Dilemmas Questionnaire- de Kogan y Wallach (1964), validado por Brockhaus (1980).

La motivación del logro de McClelland (1968) se define como el deseo por mejorar los resultados de su acción y sentirse responsables de los mismos. La necesidad de logro de McClelland va unida al concepto de Locus Control interno de Rotter (1966). Este autor determina dos aspectos de control, el interno (donde el individuo percibe el éxito o fracaso de su conducta como dependiente de sí mismo) y el externo (donde la persona no controla el resultado y lo atribuye al destino o azar). Siendo el emprendedor de éxito un individuo que tiene un autocontrol personal sobre su propio destino (Korunka, Frank, Lueger, y Mugler, 2003). De hecho en los estudios realizados con estudiantes universitarios, se establece la significación entre emprendimiento y elevados niveles de control interno (Athayde, 2009; Ismail et al., 2013; Lüthje y Franke, 2003; Robinson et al., 1991; Wang et al., 2011), pero no siempre con motivación del logro (Ismail et al., 2013; Robinson et al., 1991). En relación a los ítems para contrastar el control interno, los escogemos del formulario de la escala I-E de Rotter (1966), en la versión reformulada por Levenson (1973). Mientras que los ítems para evaluar la variable de necesidad de logro, los escogeremos de la escala de Robinson (1991), y del test ATE - Attitudes Toward Enterprise- de Athayde (2009).

En otro orden de cosas, en la construcción de los ítems que dimensionan los factores endógenos y exógenos de la percepción de viabilidad del sujeto, se ha utilizado el escalado de Likert (1932) en cuatro posiciones.

Para el análisis y parametrización de las variables exógenas de financiación y desempleo se ha tenido en cuenta los informes anuales del GEM- Global Entrepreneurship Monitor-, una organización internacional que tiene como objetivo medir el emprendimiento a nivel mundial. En el caso del desempleo y su relación con el emprendimiento, se observa en el informe GEM de 2011, un crecimiento sostenido de la tasa iniciativa emprendedora por necesidad (siendo del 15,8\% en 2008; y del 25,8\% en 2011). Y, también, un incremento de las dificultades de financiación, que se constata en una menor aportación de capital semilla a la actividad emprendedora (disminuye cada año), y a una mayor necesidad de aportación de financiación ajena (70\% de los nuevos emprendedores necesitan fondos ajenos según el GEM de 2008). La teoría de la marginación de Veciana (1999) entendida como la propensión a convertirse en empresarios aquellas personas con escasas oportunidades laborales, constata la relación existente entre desempleo y emprendimiento por necesidad (Espí et al., 2007; Linan et al., 2011). Y en relación a la falta de financiación, se percibe por el sujeto como un gran obstáculo en la viabilidad del proyecto (Espí et al., 2007; Lüthje y Franke, 2003).

En su caso, la variable esfuerzo y perseverancia va más allá del considerado sacrificio en tiempo. De hecho emprender tiene su coste de oportunidad: renunciar a un trabajo estable y seguro (Singh y DeNoble, 2003). Pero también tiene implicaciones de sacrificio a nivel económico. El emprendedor deberá comprometer recursos financieros periódicos de acuerdo a su proyecto de negocio (Hisrich y Peters, 2002). En los estudios con alumnos universitarios se constató la variable del esfuerzo como factor clave en el emprendimiento (Espí et al., 2007; Singh y DeNoble, 2003).

Una de las razones para emprender es encontrar una oportunidad, definida como cualquier actividad que requiera invertir escasos recursos a la espera de un retorno (Austin, Stevenson, y Wei-Skillern, 2006). Asimismo, también se emprende por perder el trabajo. O por deseo propio dado que se identificó una oportunidad (Korunka et al., 2003). Según Kirzner (1973) el espíritu emprendedor va estrechamente ligado al estado de alerta hacia nuevas oportunidades. Y es aquí donde interviene la variable de creatividad e innovación. Un sujeto creativo e innovador es capaz de detectar nuevas oportunidades que han pasado desapercibidas por el resto (Kirzner, 1973). Para Athayde (2009) las personas creativas son los percusores del comportamiento innovador que a su vez es clave para detectar nuevas oportunidades. La correlación entre innovación y emprendimiento es significativa, siendo especialmente fuerte como percepción de viabilidad (Ismail et al., 2013; Robinson et al., 1991; Wang et al., 2011). Los ítems escogidos en relación con la variable de creatividad e innovación son una adaptación del EAO de Robinson (1991).

Continuando con esta revisión, la autoeficacia es considerada como la percepción de control en el comportamiento (Ajzen, $\underline{1987,1991)}$ o como la percepción de viabilidad en la intención a emprender (Krueger y Carsrud, 1993; Shapero y Sokol, 1982). La autoeficacia permite también reducir los posibles errores, contratiempos y problemas que puedan surgir durante el largo proceso de emprendimiento (Bandura, 1986). Siendo además el mejor predictor al buen desempeño profesional_(Bandura, 1977, 1986; Wood y Bandura, 1989). La autoeficacia es una variable fuertemente significativa en todos los estudios con estudiantes universitarios y en relación a su potencial emprendedor (Chen et al., 1998; Krueger, Reilly y Carsrud, 2000; Linan et al., 2011; Liñán y Chen, 2009). Los ítems seleccionados son adaptados del cuestionario EIQ - Entrepreneurial Intention Questionnaire de los autores Liñan y Chen (2009).

\section{Parámetros de la muestra: los expertos y los participantes}

Una vez definidos los ítems del instrumento según los autores y bibliografía más relevantes, es conveniente analizar la validez de contenido mediante un juicio de expertos. Así y según los autores Skjong y Wentwortht (2000) los criterios que un experto debe poseer son experiencia en la realización de juicios y expertise en la temática objeto de análisis. También es determinante su grado de objetividad, de imparcialidad y de buena reputación en la comunidad.

En nuestra investigación, se han seleccionado tres expertos del grupo de investigación "Emprèn" y la responsable de la Unidad de Emprendimiento que a su vez son profesores del departamento de economía y empresa de la facultad de empresa y comunicación de la Universitat de Vic - Universitat Central de Catalunya. Asimismo, incorporamos dos emprendedores de la Agencia de Emprendimiento, Innovación y Conocimiento (Creacció). Los expertos han identificado y corregido aquellos ítems de dudosa redacción y que consideraban inadecuados para medir la credibilidad emprendedora y que contribuya a la validación del instrumento.

En lo que se refiere al tamaño muestral, nos encontramos en una proporción de 4 a 1, para las dimensiones que son explicadas por un mayor número de ítems. En concreto, el tamaño muestral supera las exigencias de Kline (1993) y queda ligeramente inferior al criterio de Nunnally (1978) que marcaría 90 sujetos, para las dimensiones con 18 ítems. Para el resto de dimensiones, 17 
en su conjunto, la proporción supera ampliamente la ratio 5 a 1 estipulado por Nunnally (1978) siendo el tamaño de la muestra el adecuado.

Finalmente, cabe apuntar que, para la selección de la muestra, se han analizado 84 estudiantes utilizando el método aleatorio por conglomerados de forma natural, donde la unidad muestral es un grupo de elementos de la población que forman una unidad (jornada del programa de emprendimiento de la universidad que se desarrolló en la UVIC-UCC en septiembre 2015.

\section{Resultados}

\section{Validez de contenido}

La validez de contenido se refiere al grado en que los ítems o puntuaciones de una escala-instrumento representan un universo temático donde la medición empírica cubre la variedad de significados que incluye un concepto (Bohrnstedt, 1976; Cea d'Ancona, 1998; Muñiz, 2005). En este sentido, la validez de contenido determina si el instrumento de medida sirve para medir el comportamiento que se pretende medir. Es por este motivo que en la construcción de las dimensiones se han utilizado ítems que previamente han sido contrastados y utilizados por otros autores de referencia (ver anexo 6.1). Los ítems también se pusieron a disposición del juicio de expertos. Los resultados del juicio indicaron que la gran mayoría de los ítems eran adecuados y bien redactados. Solamente en algunos ítems de nueva creación era necesaria alguna corrección, especialmente de comprensión y simplificación por uso de frases con doble negativo, por ejemplo.

\section{Validez de constructo}

Para determinar la validez de constructo y los índices de fiabilidad del instrumento es necesaria la prueba piloto y el posterior análisis factorial mediante la extracción de componentes principales con rotación Varimax. ${ }^{1}$ Cabe añadir que en cada conjunto de ítems que explican una variable o dimensión se ha procedido a su análisis en dos fases:
1.- Verificar si es posible aplicar el análisis factorial.

Para ello se observa la medida de adecuación muestral (KMO) que contrasta las correlaciones parciales entre las variables. El estadístico varía entre 1 y 0 siendo significativo los valores superiores a 0,5. Asimismo se ha aplicado la prueba de esfericidad de Bartlett que contrasta la hipótesis nula -la matriz de correlaciones es una matriz de identidad- en cuyo caso no existirían correlaciones significativas entre las variables y el modelo factorial no sería adecuado.

En cada uno de los casos procedemos a estudiar el negativo del coeficiente de correlación parcial, llamado coeficiente de correlación anti-imagen. Si la matriz contiene correlaciones anti-imagen, entonces nos indica que hay un elevado número de coeficientes altos que deben tomarse en cuenta antes de aplicar el análisis factorial. En la matriz de correlación anti-imagen se deben observar pocos valores elevados en términos absolutos y no debería haber un número elevado de coeficientes ceros, pues de lo contrario se recomienda no llevar a cabo el análisis factorial. Finalmente indicar que comprobamos también la factibilidad de la aplicación del análisis factorial examinando la diagonal de la matriz anti-imagen. Dicha diagonal permite ver el valor de las medidas de adecuación que presenta cada variable y que conocemos como MSA (Measure of Sampling Adecuacy). Este tipo de medida permite comprobar variable por variable si es adecuado llevar a cabo el análisis factorial. Aquí se toma como valores mínimos y máximos respectivamente el 0 y el 1, siendo tanto mejor cuanto mayor sea el valor del MSA.

2.- Corroborar si es viable el análisis factorial, analizando para ello la correlación del estadístico total-elemento.

En concreto observamos para cada uno de los ítems que pertenecen a una variable o dimensión si su correlación elemento-total corregida es lo suficientemente pequeña (indicando falta de explicación del ítem en relación con su variable o dimensión). Y, además, en caso de constatarlo, ver la variación positiva del Alfa de Cronbach en el caso de eliminar el ítem o elemento (ver Tabla 1).

Tabla 1. Ítems y fiabilidad por tipo de dimensión. Fuente: elaboración propia.

\begin{tabular}{|c|c|c|c|c|c|}
\hline \multirow[b]{2}{*}{ DIMENSIONES } & \multicolumn{2}{|c|}{ Formulario Inicial } & \multicolumn{3}{|c|}{ Formulario definitivo } \\
\hline & $\begin{array}{c}\text { No }^{\circ} \\
\text { Items }\end{array}$ & $\begin{array}{c}\text { Alfa } \\
\text { Cronbach }\end{array}$ & $\begin{array}{c}\text { No }^{\circ} \\
\text { Items }\end{array}$ & $\begin{array}{c}\text { Alfa } \\
\text { Cronbach }\end{array}$ & Fiabilidad \\
\hline Potencial emprendedor & 5 & 0,881 & 5 & $0,0,881$ & Buena \\
\hline Neuroticismo & 18 & 0,763 & 13 & 0,802 & Buena \\
\hline Extraversión & 18 & 0,693 & 14 & 0,734 & Aceptable \\
\hline Apertura & 18 & 0,631 & 15 & 0,684 & Aceptable \\
\hline Amabilidad & 18 & 0,782 & 16 & 0,794 & Buena \\
\hline Responsabilidad & 18 & 0,82 & 16 & 0,843 & Buena \\
\hline Autoestima & 5 & 0,272 & 2 & 0,494 & Suficiente \\
\hline Automonitoreo & 5 & 0,603 & 4 & 0,659 & Aceptable \\
\hline Propensión al riesgo & 5 & 0,455 & 4 & 0,523 & Suficiente \\
\hline Locus control & 5 & 0,455 & 4 & 0,523 & Suficiente \\
\hline Motivación del logro & 5 & 0,596 & 3 & 0,57 & Suficiente \\
\hline Financiación & 5 & 0,58 & 4 & 0,603 & Aceptable \\
\hline Desempleo y ciclo económico & 4 & 0,72 & 2 & 0,844 & Buena \\
\hline Esfuerzo personal y perseverancia & 4 & 0,301 & 3 & 0,417 & Regular \\
\hline Creatividad e innovación & 4 & 0,454 & 3 & 0,51 & Suficiente \\
\hline Autoeficacia & 4 & 0,876 & 4 & 0,876 & Buena \\
\hline Propensión por actuar & 2 & $-0,163$ & 0 & 0 & Nula/eliminar \\
\hline Total, ítems formulario & 143 & & 111 & & \\
\hline
\end{tabular}

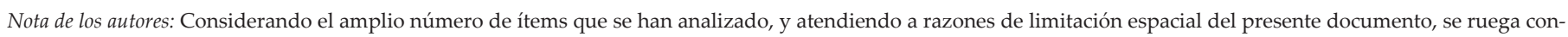

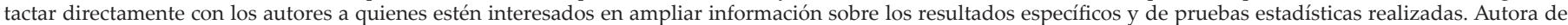
correspondencia: elisenda.tarrats@uvic.cat. 
En la mayoría de las dimensiones ha sido posible realizar su análisis factorial, excepto en la variable de propensión a actuar, debido a un determinante muy cercano a uno $(0,994)$ y la aceptación de la hipótesis de matriz de identidad en la prueba de esfericidad de Barlett (sig.: 0,496) que nos obliga a eliminar del cuestionario la dimensión propensión a actuar y sus correspondientes ítems.

Como criterio general, George y Mallery (2003) consideran coeficientes fiables los alfa de cronbach superiores a 5. Siendo el rango de 5 a 6 suficiente, pero "pobre". En nuestro caso existe una variable por debajo de 5 que es "esfuerzo y perseverancia". Esto indicaría que el análisis de los estudiantes en este factor debe hacerse con cautela y evaluando los resultados sabiendo que pueden ser poco fiables. Para el resto, y una vez eliminada la variable propensión a actuar, se concluye la disponibilidad de un cuestionario fiable, cuyos resultados pueden ser analizados asumiendo la validez de contenido y de constructo.

El análisis factorial concluye que reduciendo los ítems de 143 a 111 se mejora la fiabilidad de todas las dimensiones a excepción de la variable dependiente de potencial emprendedor y la variable independiente de autoeficacia que inicialmente ya contaban con una alta fiabilidad. Es importante destacar que las distintas dimensiones son variables independientes que intentan explicar a la variable dependiente de potencial emprendedor. Es por ello que observar la correlación entre ellas, y especialmente con credibilidad emprendedora nos puede dar indicaciones sobre como son los sujetos emprendedores y sobretodo saber si ser emprendedor puede aprenderse o es una característica de carácter más innato.

\section{Discusiones y conclusiones}

La construcción del cuestionario definitivo de medición de la credibilidad emprendedora nos permitirá su uso y posterior análisis, en cualquier de los ámbitos de la educación superior postobligatoria. Cabe señalar que observando las correlaciones con la variable dependiente de potencial emprendedor podremos prever si el emprendimiento puede ser desarrollado y aprendido mediante actividades formativas, o, por lo contrario, es innato y/o depende de ajenas circunstancias.

En la muestra utilizada en la prueba piloto ya hemos constatado algunos resultados significativos de variables que inciden significativamente con el potencial emprendedor. Estos resultados se han analizado mediante la matriz de coeficientes de correlación (Rho Spearman) y mediante el uso de las pruebas de U de Mann-Whitney y $\mathrm{W}$ de Wilcoxon en las dimensiones nominales dicotómicas. El género masculino, tener familiares empresarios, disponer de experiencia laboral previa y haber cursado cursos sobre creación de empresas inciden significativamente, en correlación positiva, con el potencial emprendedor. En cambio, incide, pero en signo contrario el neuroticismo. Por otro lado, destacamos que ejercen más influencia aquellas variables relacionadas con la percepción de viabilidad. Siendo la autoeficacia, creatividad e innovación, desempleo y esfuerzo y perseverancia las variables que más correlacionan en intensidad y significación con el potencial emprendedor. Esto significa que un sujeto que sea capaz de detectar nuevas oportunidades muestre un elevado desempeño (sabe lo que tiene que hacer), es perseverante, y está dispuesto a sacrificarse en tiempo y dinero, tiene un elevado potencial emprendedor.

Ahora bien, también es importante relacionar los efectos indirectos. Es decir, analizar qué variables afectan a las mencionadas. En este sentido observamos que es la falta de financiación la que más incide con la autoeficacia. Y en el caso de la creatividad e innovación son las variables de motivación del logro, responsabilidad, apertura y propensión al riesgo las que mayor efecto directo ejercen.

En conclusión, estos datos extraídos de la prueba piloto nos permitirán disponer de un cuestionario fiable, salvo para la dimensión de esfuerzo y perseverancia que obtiene una fiabilidad insuficiente.

\section{Referencias bibliográficas}

Ajzen, I. (1987). Attitudes, traits, and actions: Dispositional prediction of behavior in social psychology. Advances in Experimental Social Psychology, 20, 1-63.

Ajzen, I. (1991). The theory of planned behavior. Organizational Behavior and Human Decision Processes, 50, 179-211.

Athayde, R. (2009). Measuring Enterprise Potential in Young People. Entrepreneurship Theory and Practice, 481-500.

Austin, J., Stevenson, H., y Wei-Skillern, J. (2006). Social and Commercial Entrepreneurship: Same, Different, or Both? Entrepreneurship Theory and Practice, 30(1), 1-22. DOI: https:// doi.org/10.1111/j.1540-6520.2006.00107.x

Bandura, A. (1977). Social Learning Theory. Englewood Cliffs, NJ: Prentice Hall.

Bandura, A. (1986). The Social Foundations of Thought and Action. Englewood Cliffs, NJ: Prentice-Hall.

Bohrnstedt, G. W. (1976). Evaluación de la confiabilidad y validez en la medición de actitudes. México: G.F. Summe.

Branden, N. (1993). El poder de la autoestima. Cómo potenciar este importante recurso psicológico. Buenos Aires: Editorial Paidós.

Brice, J. (2002). The role of personality dimensions on the formation of entrepreneurial intentions. Mississipi State University Press.

Brockhaus, R. H. (1980). Risk taking propensity of entrepreneurs. Academy of Management Journal, 23(3), 509-520.

Carayannis, E. G., Evans, D., y Hanson, M. (2003). A cross-cultural learning strategy for entrepreneurship education: outline of key concepts and lessons learned from a comparative study of entrepreneurship students in France and the US. Technovation, 23(9). DOI: https://doi.org/10.1016/S01664972(02)00030-5

Cea d'Ancona, M. A. (1998). Metodología cuantitativa. Estrategias y técnicas de investigación social. Madrid: Síntesis.

Chen, C. C., Greene, P. G., y Crick, A. (1998). Does entrepreneurial self-efficacy distinguish entrepreneurs from managers? Journal of Business Venturing, 13, 295-316.

Costa, P. T., y Mc Crae, R. R. (2002). Inventario de personalidad Neo Revisado (NEO-PI-R). Madrid: TEA Ediciones.

Crant, J. M. (1996). The proactive personality scale as a predictor of entrepreneurial intentions. Journal of Small Business Management, 34(3), 42-49.

Drost, E. (2010). Entrepreneurial intentions of business students in Finland: implication for education. Advances in Management, 3(7), 28-35.

Dyer, W. G. J. (1994). Toward a theory of entrepreneurial careers. Entrepreneurship Theory and Practice, 19(19), 7-21.

Eldelman, L., Manolova, T., y Brush, C. (2008). Entrepreneurship Education: Correspondence between Practices of Nascent entrepreneurs and textbook prescriptions for success. Academy of Management Learning and Education, 7(1), 56-70.

Espí, M. T., Arana, G., Heras, I., y Diaz, A. (2007). Perfil emprendedor del alumnado universitario del campus de Gipuzkoa de la Upv/Ehu. Revista de Dirección y Administración de Empresas, 14, 83-110.

Europeo, Consejo (2000). Estrategia de Lisboa. Consejo Europeo. Lisboa: Consejo Europeo. 
Eurostat (2009). Eurostat statistical book. European Union: Publication Office.

Eurostat (2014). Eurostat regional yearbook 2014. (European U). Eurostat Statistical Books. DOI: https://doi.org/10.2785/54659

Eysenck, H. J. (1970). Fundamentos biológicos de la personalidad. Barcelona: Fontanella.

Eysenck, H. J., y Eysenck, S. B. G. (1994a). EPI : Cuestionario de Personalidad EPI : manual. Madrid: TEA.

Eysenck, H. J., y Eysenck, S. B. G. (1994b). EPI : cuestionario de personalidad EPI : manual. Madrid: TEA.

George, D., y Mallery, P. (2003). SPSS for Windows step by step: a Simple Guide and Reference. Boston: A. y Bacon.

Harvey, S., y Keashly, L. (2003). Predicting the risk for agression in the workplace: Risk factors, self-esteem and time at work. Social Behavior and Pesonality: An International Journal, 31(8), 807-814.

Henry, C., Hill, F., y Leitch, C. (2005). Entrepreneurship education and training: can entrepreneurship be taught? Part I. Education+Training, 47(2), 98-111.

Hisrich, R., y Peters, M. (2002). Entrepreneurship (Vol. 5). Nueva York: Mc Graw Hill.

Honig, B. (2004). Entrepreneurship Education: Toward a Model of Contingency-Based Business Planning. Academy of Management Learning y Education, 3(3), 258-273. DOI: https://doi. org/10.5465/AMLE.2004.14242112

Honig, B., y Davidsson, P. (2003). The role of social and human capital among nascent entrepreneurs. Journal of Business Venturing, 18, 301-331.

Horney, K. (1945). Our inner conflicts (W.W Norton). New York.

Ismail, N., Jaffar, N., y Siow, T. (2013). Using EAO model to predict the self-employment intentions among the Universities' Undergraduates in Malaysia. International Journal of Trade, Economics and Finance, 4, 282-287.

Jose, F., Liñan, F., y Santos, J. (2009). Cognitive Aspects of Potential Entrepreneurs in Southern and Northern Europe: an Analysis Using GEM-Data. Revista de Economía Mundial, 23, 151-178.

Kirzner, I. M. (1973). Competition and entrepreneurship. Chicago: The University of Chicago Press.

Kline, P. (1993). The handbook of psychological Testing. London: Routledge.

Kogan, N., y Wallach, M. A. (1964). Risk taking: A study in cognition and personality. New York: W. Holt, Rinehart.

Kolvereid, L. (1996). Prediction of employment status choice intentions. Entrepreneurship Theory and Practice, 21(1), 47-57.

Korunka, C., Frank, H., Lueger, M., y Mugler, J. (2003). The Entrepreneurial Personality in the Context of Resources, Environment, and the Startup Process?A Configurational Approach. Entrepreneurship Theory and Practice, 28(1), 23-42. DOI: https://doi.org/10.1111/1540-8520.00030

Krauss, C. (2011). Actitudes emprendedoras de los estudiantes universitarios: El caso de la Universidad Católica del Uruguay. Dimens empres, 9(1), 28-40.

Krueger, N. F., y Carsrud, A. L. (1993). Entrepreneurial intentions: applying the theory of planned behavior. Entrepreneurship and Regional Development, 5(4), 315-330.

Krueger, N. F., Reilly, M. D., y Carsrud, A. L. (2000). Competing models of entrepreneurial intentions. Journal of Business Venturing, 15(5-6), 411-432. DOI: https://doi.org/10.1016/S08839026(98)00033-0

Krueger Jr, N. F., y Brazeal, D. V. (1994). Entrepreneurial Potential and Potential Entrepreneurs. Entrepreneurship Theory and Practice, 91-104.

Lennox, R. D., y Wolf, R. N. (1984). Revision of the Self-Monitoring Scale. Journal of Personality and Social Psychology, 1361.
Lepoutre, J., Berghe, W. Van den, Tilleuil, O., y Crijns, H. (2010). A new approach to testing the effects of entrepreneurship education among secondary school pupils. Vlerick Leuven Gent Management School Working Paper Series.

Levenson, H. (1973). Multidimensional locus of control in psychiatric patients. Journal of Consulting and Clinical Psychology, 41, 397-404.

Likert, R. (1932). A technique for the Measurement of Attitudes. Archives of Psychology, 140, 44-53.

Liles, P. R. (1974). New business ventures and entrepreneur. Homewood, Illinois: Richard D. Irwin.

Linan, F., Urbano, D., y Guerrero, M. (2011). Regional variations in entrepreneurial cognitions: Start-up intentions of university students in Spain. Entrepreneurship and Regional Development, 23(3-4). DOI: https://doi.org/10.1080/08985620903233929

Liñán, F., y Chen, Y.-W. (2009). Development and Cross-Cultural Application of a Specific Instrument to Measure Entrepeneurial Intentions. Entrepreneurship Theory and Practice, 593-617.

Liñán, F., Santos, F. J., y Fernández, J. (2011). The influence of perceptions on potential entrepreneurs. International Entrepreneurship and Management Journal, 7(3), 373-390.

Lounsbury, J. ., Gibson, L. ., Sundstrom, E., Wilburn, D., y Loveland, J. M. (2004). An empirical investigation of the proposition that "school is work": a comparison of personality-performance in school and work settings. Journal of Education and Work, 17(1), 119-131.

Lüthje, C., y Franke, N. (2003). The "making" of an entrepreneur: testing a model of entrepreneurial intent among engineering students at MIT. RyD Management, 33(2), 135-147.

Mc Clelland, D. C. (1968). La sociedad ambiciosa. Factores psicológicos en el desarrollo económico. Madrid: Guadarrama.

McCrae, R., y Costa, P. (2003). Personality in adulthood: a five-factor theory perspective. New York: Guilford.

Muñiz, J. (2005). Utilización de los tests. Madrid: La Muralla.

Nandamuri, P. P., Gajulapally, R. K., y Gowthami, C. H. (2012). A Strategic Analysis of Entrepreneurial Orientation of Management Graduates. The IUP Journal of Business Strategy, 9(4), 7-23.

Nunnally, J. C. (1978). Psychometric theory. New York: Mc Graw-Hill.

Peterman, N. E., y Kennedy, J. (2003). Enterprise Education: Influencing Students Perceptions of Entrepreneurship. Entrepreneurship Theory and Practice, 28(2), 129-144.

Piaget, J. (1995). The essential Piaget. New York: Voneche y H. Gruber.

Reichert, S. (2009). Institutional diversity in European higher education. Zurich: European University Association.

Robbins, S. P. (1996). Comportamiento organizacional: Teoria y práctica (vol. 7). Mexico: Prentice Hall.

Robinson, P. B., Stimpson, D. V, Huefner, J. C., y Hunt, H. K. (1991). An attitude approach to the prediction of entrepreneurship. Entrepreneurship Theory and Practice, 15(4), 13-31.

Rotter, J. B. (1966). Generalized Expectancies for Internal versus External Control of reinforcement. Psychological Monographs, Whole number 609, 80(1), 1-28.

Rusell, R., Atchison, M., y Brooks, R. (2008). Business plan competitions in tertiary institutions: encouraging entrepreneurship education. Journal of Higher Education Policy y Management, 30(2), 123-138.

Russell, M. T., Cattell, R. B., Cattell, A. K. S., Cattell, H. E. P., Karol, D. L., y Seisdedos Cubero, N. (1997a). 16 PF-5 : manual. Madrid : TEA. 
Russell, M. T., Cattell, R. B., Cattell, A. K. S., Cattell, H. E. P., Karol, D. L., y Seisdedos Cubero, N. (1997b). 16 PF-5 : manual. Madrid: TEA.

Shapero, A., y Sokol, L. (1982). The social dimension of entrepreneurship. (C. A. Kent, D. L. Sexton, y K. H. Vesper (Eds.). The encyclopedia of entrepreneurship. Englewood Cliffs, NJ: Prentice Hall.

Singh, G., y DeNoble, A. (2003). Views on self-employment and personality: an exploratory study. Journal of Developmental Entrepreneurship, 8(3), 265-281.

Skjong, R., y Wentworth, B. (2000). Expert Judgement and risk perception. Norway: Det Norske Veritas.

Souitaris, V., Zerbinati, S., y Al-Laham, A. (2007). Do entrepreneurship programmes raise entrepreneurial intention of science and engineering students? The effect of learning, inspiration and resources. Journal of Business Venturing, 22(4). DOI: https://doi.org/10.1016/j.jbusvent.2006.05.002

Thursby, M., Fuller, A., y Thursby, J. (2009). An integrated approach to educating professionals for carees in innovation. Academy of Management Learning y Education, 8(3), 389-405.
UNESCO. (2004). Compendio mundial de la educación. Montreal: Instituto de Estadística de la UNESCO.

Veciana, J. M. (1999). Creación de empresas como programa de investigación científica. Revista Europea de Dirección Y Economía de La Empresa, 8(3), 11-36.

Veciana, J. M., Aponte, M., y Urbano, D. (2005). “University students" attitudes towards entrepreneurship: A two countries comparison. The International Entrepreneurship and Management, 1(2), 165-182.

Wang, C. K., y Wong, P. K. (2004). Entrepreneurial interest of university students in Singapore. Technovation, 24(2). doi: https://doi.org/10.1016/S0166-4972(02)00016-0

Wang, W., Lu, W., y Millington, J. K. (2011). Determinants of Entrepreneurial Intention among College Students in China and USA. Journal of Global Entrepreneurship Research, 1(1), 35-44.

Wood, R., y Bandura, A. (1989). Social cognitive theory of organizational management. Academy of Management Review, 14, 361-384. 